\title{
Nurses' perceptions of the sustainability of a standardised assessment for preventing complications in a ICU: a qualitative study
}

\begin{abstract}
Background: Quality improvement projects have been widely adopted to prevent complications in the ICU. Objective: This paper describes nurses' perceptions of implementation strategies and the potential sustainability of a practice change intervention to prevent complications in a Malaysian ICU. Design: A participatory action research using five focus group discussions were undertaken with 19 nurses in a single ICU in regional Malaysia. Focus group transcripts were analysed using thematic analysis. Results: The main themes derived from the interviews were: [1] Empowering staff to embrace evidence-based practices; [2] Staff knowledge, attitudes, and beliefs that impact on behaviour; and [3] management support and leadership are influential in staff behaviours (acceptance \& perseverance of change process). Discussion: Resistance to change was recognized as a barrier to adopting evidence based recommendations. There is a need to improve nurses' knowledge, attitude and awareness of the importance of assessment for VAP, CRBSI and PIs in the ICU.
\end{abstract}

Keyword: Malaysia; Clinical practice improvement; Intensive care unit; Qualitative study 